\title{
Indicadores pronósticos en el tromboembolismo pulmonar
}

Calvo Romero JM, Lima Rodríguez. EM. Indicadores pronósticos en el tromboembolismo pulmonar. An Med Interna (Madrid) 2008; 25: 1-3.

\section{INTRODUCCIÓN}

El pronóstico del tromboembolismo pulmonar (TEP) varía ampliamente (1). Podemos enfrentarnos a distintas situaciones que van desde un TEP masivo potencialmente fatal a un TEP periférico con escasa repercusión. En este artículo revisamos los indicadores pronósticos de eventos adversos, especialmente de mortalidad, en pacientes con TEP.

\section{INDICADORES CLÍNICOS Y DE LABORATORIO BÁSICOS}

Los pacientes con TEP con shock y/o parada cardíaca tienen una mortalidad muy elevada que se ha estimado en el 58$65 \%(2,3)$. Además existen otras características clínicas que conllevan un mal pronóstico. En el registro RIETE, se ha descrito que los pacientes sin enfermedad cardiopulmonar previa en los que el TEP se presenta como un síndrome de infarto pulmonar tienen una mortalidad a los 3 meses menor que los pacientes con los síndromes de disnea aislada o colapso circulatorio (4). En el registro ICOPER, una edad superior a 70 años y la presencia de cáncer, insuficiencia cardíaca, enfermedad pulmonar obstructiva crónica, taquipnea y/o hipotensión arterial sistólica se asociaron a un aumento de la mortalidad a los 3 meses (3).

Wicki y cols. (5) describieron un modelo para predecir el riesgo a los 3 meses de eventos adversos (muerte, recurrencia y/o hemorragia grave) en pacientes con TEP. En este modelo se asigna una puntuación a una serie de datos: cáncer (2 puntos), presión arterial sistólica inferior a $100 \mathrm{mmHg}$ (2 puntos), insuficiencia cardíaca (1 punto), presión arterial de oxígeno basal inferior a $60 \mathrm{mmHg}$ (1 punto), trombosis venosa profunda (TVP) en la ecografía-doppler venosa de miembros inferiores (1 punto) y antecedente de TVP (1 punto). La frecuencia de eventos adversos en los pacientes con una puntuación total igual o inferior a 2 fue del $2 \%$ y en aquellos con una puntuación total superior a 2 del 26\%. Este modelo ha sido posteriormente validado con un valor predictivo negativo para eventos adversos del 95\% (6), y del
$92,6 \%$ en un pequeño estudio en nuestro medio (7). Se han descrito otros modelos más complicados, que incluyen fundamentalmente variables clínicas, capaces de predecir la mortalidad y otros eventos adversos en el TEP (8).

En un pequeño estudio de pacientes con TEP sin enfermedad pulmonar crónica, un gradiente alvéolo-arterial de oxígeno superior a $53 \mathrm{mmHg}$ tuvo un valor predictivo negativo del 92\% para mortalidad a los 30 días (9).

\section{MARCADORES CARDIACOS}

Las troponinas I y T son marcadores sensibles y específicos de daño miocárdico. Según la población estudiada, un porcentaje variable de pacientes con TEP presenta elevación de las troponinas, que se ha atribuido a isquemia miocárdica de las cavidades derechas (10-19). Esta elevación es considerablemente más frecuente en los pacientes con disfunción ventricular derecha (10-16). La mortalidad está aumentada en pacientes con TEP y niveles de troponina I o troponina T superiores a $0,5-1,5 \mathrm{ng} / \mathrm{ml}$ y $0,1 \mathrm{ng} / \mathrm{ml}$, respectivamente $(11,12,14,15)$. En un estudio de 458 pacientes con TEP submasivo, definido por la existencia de disfunción ventricular derecha con presión arterial sistólica superior a $90 \mathrm{mmHg}$, una troponina I superior a $0,5 \mathrm{ng} / \mathrm{ml}$ se asoció a un aumento de 3,5 veces del riesgo de muerte a los 3 meses (14). La cinética de liberación de las troponinas parece ser distinta en el TEP y en el síndrome coronario agudo, al permanecer elevadas menos tiempo en los pacientes con TEP $(17,18)$. Las troponinas I y T se suelen normalizar tras las primeras 72 horas del inicio de los síntomas del TEP $(17,18)$.

Los péptidos natriuréticos $\mathrm{B}$ y pro-B también tienen valor pronóstico en el TEP. Su elevación se asocia a la existencia de disfunción ventricular derecha (20). Un péptido natriurético $\mathrm{B}$ o pro-B normal tiene un valor predictivo negativo para eventos adversos del 93-97\% $(21,22)$. El nivel de péptido natriurético $\mathrm{B}$ se asocia a la ocurrencia de TEP fatal o recurrencia en el seguimiento a 3 meses de pacientes en TEP hemodinámicamente estables (23). Recientemente 
se ha descrito que la normalidad de la troponina $\mathrm{T}$ y del péptido natriurético pro-B en pacientes con TEP se asocia a un excelente pronóstico $(24,25)$.

\section{DISFUNCIÓN VENTRICULAR DERECHA}

Aproximadamente hasta la mitad de los pacientes con TEP y presión arterial sistólica normal puede tener evidencia ecocardiográfica de disfunción ventricular derecha (26). Los criterios ecocardiográficos habitualmente utilizados para definir la disfunción ventricular derecha son la hipoquinesia y dilatación ventricular derecha y la hipertensión pulmonar (26). Reuniendo los resultados de 4 estudios prospectivos, la mortalidad a corto plazo relacionada con el TEP en pacientes normotensos fue del 9,3\% en los que presentaron disfunción ventricular derecha ecocardiográfica frente al 0,4\% en los que no la presentaron (26). En el registro ICOPER, aproximadamente el $40 \%$ de 1035 pacientes con TEP y presión arterial sistólica superior a $90 \mathrm{mmHg}$ con valoración ecocardiográfica presentaron hipoquinesia ventricular derecha (27). La mortalidad a los 3 meses fue significativamente superior en los que presentaron hipoquinesia ventricular derecha que en los que no la presentaron, $16,3 \%$ frente a $9,4 \%$ (27). Los pacientes con TEP de un pequeño estudio con troponina I y ecocardiograma normales tuvieron una supervivencia a los 3 meses del $98 \%$ (28). Se ha propuesto un algoritmo para estratificar el riesgo en los pacientes con TEP que comenzaría con la determinación de marcadores cardíacos (troponinas y/o péptidos natriuréticos), seguido de ecocardiograma si éstos están elevados (29).

La existencia de una presión arterial sistólica pulmonar superior a $50 \mathrm{mmHg}$ calculada mediante ecocardiograma al diagnóstico del TEP se asocia a un mayor riesgo de hipertensión pulmonar persistente y posiblemente de muerte a largo plazo (30). En pacientes con TEP con disfunción ventricular derecha, la presencia de un foramen oval permeable detectado mediante ecocardiograma de contraste parece acompañarse de un aumento de la mortalidad intrahospitalaria (31). Igualmente, la detección ecocardiográfica de trombos flotantes en corazón derecho conlleva una elevada mortalidad (32). Los pacientes con TEP y persistencia de disfunción ventricular derecha ecocardiográfica al alta tienen un riesgo aumentado de recurrencias (33).

La disfunción ventricular derecha también parece poder valorarse mediante tomografía computarizada. La dilatación ventricular derecha, cociente de los diámetros del ventrículo derecho y ventrículo izquierdo superior a 0,9-1, detectada mediante tomografía computarizada en el momento del diagnóstico del TEP se ha asociado a un aumento de la mortalidad en los primeros meses en algunos estudios $(34,35)$. No obstante, el mismo criterio en otro estudio no se asoció a un aumento de la mortalidad en los 30 primeros días (36).

\section{OTROS INDICADORES PRONÓSTICOS}

El electrocardiograma también tiene valor pronóstico en el TEP. La presencia de una onda $\mathrm{T}$ negativa en la derivaciones precordiales se ha asociado a una mayor severidad del TEP (37). La presencia de una onda T negativa en 7 o más derivaciones se asoció a un riesgo aumentado de complicaciones precoces en un pequeño estudio de pacientes con TEP (38). Un patrón Qr en la derivación V1 se correlaciona con la existencia de disfunción ventricular derecha y es un indicador pronóstico de eventos adversos (39). En un gran registro prospectivo, la presencia de alteraciones electrocardiográficas como arritmias auriculares o bloqueo completo de rama derecha se asoció a un aumento de la mortalidad intrahospitalaria (40). En nuestro medio, una combinación de alteraciones electrocardiográficas (taquicardia sinusal, taquicardias supraventriculares, patrón S1Q3T3, onda T negativa en derivaciones precordiales, bloqueo de rama derecha $\mathrm{y} / \mathrm{u}$ onda $\mathrm{P}$ pulmonar) fue significativamente más frecuente en los pacientes con TEP e insuficiencia respiratoria o hipotensión arterial (41).

Existe controversia sobre si la extensión del defecto de perfusión en la gammagrafia pulmonar se correlaciona con la presencia de disfunción ventricular derecha, y por tanto puede tener valor pronóstico (42-44). No obstante, un estudió demostró que una obstrucción vascular superior al $50 \%$ valorada mediante gammagrafía pulmonar de perfusión conlleva un aumento de 8 veces de la mortalidad precoz en el TEP (45).

Clásicamente se han utilizado métodos como el índice de Miller para valorar la gravedad del TEP en base a los hallazgos en la arteriografía pulmonar (46). Un índice de obstrucción vascular basado en los hallazgos en la angio-tomografía computarizada se ha asociado a un aumento de la mortalidad en los 3 primeros meses (35). El dímero-D puede tener también valor pronóstico. Un dímero-D inferior a $1.500 \mathrm{mcg} / \mathrm{l} \mathrm{se}$ ha asociado a una muy baja mortalidad a los 3 meses en el TEP (47).

\section{J. M. CALVO ROMERO, E. M. LIMA RODRÍGUEZ}

Servicio de Medicina Interna. Hospital Ciudad de Coria. ${ }^{1}$ Medicina familiar y Comunitaria. Área de Salud. Coria, Cáceres 


\section{Bibliografía}

1. Goldhaber SZ. Pulmonary embolism. Lancet 1999; 363: 1295-305.

2. Kasper W, Konstantinides S, Geibel A, Olschewski M, Heinrich F, Grosser $\mathrm{KD}$, et al. Management strategies and determinants of outcome in acute major pulmonary embolism: Results of a multicenter registry. J Am Coll Cardiol 1997; 30: 1165-71.

3. Goldhaber SZ, Visani L, De Rosa M. Acute pulmonary embolism: Clinical outcomes in the International Cooperative Pulmonary Embolism Registry (ICOPER). Lancet 1999; 353: 1386-9.

4. Lobo JL, Zorrilla V, Aizpuru F, Uresandi F, García-Bragado F, Conget F, et al. Clinical syndromes and clinical outcome in patients with pulmonary embolism: Findings from the RIETE registry. Chest 2006; 130: 1817-22.

5. Wicki J, Perrier A, Perneger TV, Bounameaux H, Junod AF. Predicting adverse outcome in patients with acute pulmonary embolism: A risk score. Thromb Haemost 2000; 84: 548-52.

6. Nendaz MR, Bandelier P, Aujesky D, Cornuz J, Roy PM, Bounameaux H, et al. Validation of a risk score identifying patients with acute pulmonary embolism, who are at low risk of clinical adverse outcome. Thromb Haemost 2004; 91: 1232-6.

7. Calvo Romero JM, Lima Rodríguez EM. Validación de un método de predicción del riesgo de evolución desfavorable en pacientes con tromboembolia pulmonar. Arch Bronconeumol 2006; 42: 310.

8. Aujesky D, Roy PM, Le Manach CP, Verschuren F, Meyer G, Obrosky DS, et al. Validation of a model to predict adverse outcomes in patients with pulmonary embolism. Eur Heart J 2006; 27: 476-81.

9. Hsu JT, Chu CM, Chang ST, Cheng HW, Cheng NJ, Ho WC, Chung CM. Prognostic role of alveolar-arterial oxygen pressure difference in acute pulmonary embolism. Circ J 2006; 70: 1611-6.

10. Meyer T, Binder L, Hruska N, Luthe H, Buchwald AB. Cardiac troponin I elevation in acute pulmonary embolism is associated with right ventricular dysfunction. J Am Coll Cardiol 2000; 36: 1632-6.

11. Giannitsis E, Muller-Bardorff M, Kurowski V, Weidtmann B, Wiegand U, Kampmann M, et al. Independent prognostic value of cardiac troponin $\mathrm{T}$ in patients with confirmed pulmonary embolism. Circulation 2000; 102: 211-7.

12. Konstantinides S, Geibel A, Olschewski M, Kasper W, Hruska N, Jackle S, et al. Importance of cardiac troponins I and $\mathrm{T}$ in risk stratification of patients with acute pulmonary embolism. Circulation 2002; 106: 1263-8.

13. Mehta NJ, Jani K, Khan IA. Clinical usefulness and prognostic value of elevated cardiac troponin I levels in acute pulmonary embolism. Am Heart J 2003; 145: 821-5.

14. Douketis JD, Leeuwenkamp O, Grobara P, Johnston M, Sohne M, Ten Wolde $\mathrm{M}$, et al. The incidence and prognostic significance of elevated cardiac troponins in patients with submassive pulmonary embolism. J Thromb Haemost 2005; 3: 508-13.

15. La Vecchia L, Ottani F, Favero L, Spadaro GL, Rubboli A, Boanno C, et al. Increased cardiac troponin I on admission predicts in-hospital mortality in acute pulmonary embolism. Heart 2004; 90: 633-7.

16. Aksay E, Yanturali S, Kiyan S. Can elevated troponin I levels predict complicated clinical course and inhospital mortality in patients with acute pulmonary embolism? Am J Emerg Med 2007; 25: 138-43.

17. Muller-Bardorff M, Weidtmann B, Giannitsis E, Kurowski V, Katus HA. Release kinetics of cardiac troponin $\mathrm{T}$ in survivors of confirmed severe pulmonary embolism. Clin Chem 2002; 48: 673-5.

18. Punukollu G, Khan IA, Gowda RM, Lakhanpal G, Vasavada BC, Sacchi TJ. Cardiac troponin I release in acute pulmonary embolism in relation to the duration of symptoms. Int J Cardiol 2005; 99: 207-11.

19. Calvo Romero JM. Troponina I en pacientes con tromboembolismo pulmonar. An Med Interna 2005; 22: 589-90.

20. Kruger S, Graf J, Merx MW, Koch KC, Kunz D, Hanrath P, et al. Brain natriuretic peptide predicts right heart failure in patients with acute pulmonary embolism. Am Heart J 2004; 147: 60-5.

21. Kucher N, Printzen G, Doernhoefer T, Windecker S, Meier B, Hess OM Low pro-brain natriuretic peptide levels predict benign clinical outcome in acute pulmonary embolism. Circulation 2003; 107: 1576-8.

22. Kucher N, Printzen G, Goldhaber SZ. Prognostic role of brain natriuretic peptide in acute pulmonary embolism. Circulation 2003; 107: 2545-7.

23. Sohne M, Ten Wolde M, Boomsma F, Reitsma JB, Douketis JD, Buller HR Brain natriuretic peptide in hemodynamically stable acute pulmonary embolism. J Thromb Haemost 2006; 4: 552-6.

24. Styczynski G, Kuch-Wocial A, Abramczyk P, Bartoszewicz Z, Berent H, Kuczynska K. Biomarker-based risk assessment model in acute pulmonary embolism. Eur Heart J 2005; 26: 2166-72.

25. Tulevski II, ten Wolde M, van Veldhuisen DJ, Mulder JW, van der Wall EE, Buller HR, et al. Combined utility of brain natriuretic peptide and cardiac tro- ponin $\mathrm{T}$ may improve rapid triage and risk stratification in normotensive patients with pulmonary embolism. Int J Cardiol 2007; 116: 161-6.

26. Kreit JW. The impact of right ventricular dysfunction on the prognosis and therapy of normotensive patients with pulmonary embolism. Chest 2004; 125: $1539-45$

27. Kucher N, Rossi E, De Rosa M, Goldhaber SZ. Prognostic role of echocardiography among patients with acute pulmonary embolism and a systolic arterial pressure of $90 \mathrm{~mm} \mathrm{Hg}$ or higher. Arch Intern Med 2005; 165: 1777 81 .

28. Kucher N, Wallmann D, Carone A, Windecker S, Meier B, Hess OM. Incremental prognostic value of troponin I and echocardiography in patients with acute pulmonary embolism. Eur Heart J 2003; 24: 1651-6.

29. Binder L, Pieske B, Olschewski M, Geibel A, Klostermann B, Reiner C, et al. N-terminal pro-brain natriuretic peptide or troponin testing followed by echocardiography for risk stratification of acute pulmonary embolism. Circulation 2005; 112: 1573-9.

30. Ribeiro A, Lindmarker P, Johnsson H, Juhlin-Dannfelt A, Jorfeldt L. Pulmonary embolism: one-year follow-up with echocardiography doppler and fiveyear survival analysis. Circulation 1999; 99: 1325-30.

31. Konstantinides S, Geibel A, Kasper W, Olschewski M, Blumel L, Just H. Patent foramen ovale is an important predictor of adverse outcome in patients with major pulmonary embolism. Circulation 1998;97:1946-51.

32. Chartier L, Bera J, Delomez M, Asseman P, Beregi JP, Bauchart JJ, et al. Free-floating thrombi in the right heart: diagnosis, management, and prognostic indexes in 38 consecutive patients. Circulation 1999; 99: 2779-83.

33. Grifoni S, Vanni S, Magazzini S, Olivotto I, Conti A, Zanobetti M, et al. Association of persistent right ventricular dysfunction at hospital discharge after acute pulmonary embolism with recurrent thromboembolic events. Arch Intern Med 2006; 166: 2151-6.

34. Schoepf UJ, Kucher N, Kipfmueller F, Quiroz R, Costello P, Goldhaber SZ. Right ventricular enlargement on chest computed tomography: A predictor of early death in acute pulmonary embolism. Circulation 2004; 110: 327680.

35. van der Meer RW, Pattynama PM, van Strijen MJ, van den Berg-Huijsmans AA, Hartmann IJ, Putter H, et al. Right ventricular dysfunction and pulmonary obstruction index at helical CT: Prediction of clinical outcome during 3month follow-up in patients with acute pulmonary embolism. Radiol 2005; 235: 798-803.

36. Araoz PA, Gotway MB, Harrington JR, Harmsen WS, Mandrekar JN. Pulmonary embolism: Prognostic CT findings. Radiol 2007; 242: 889-97.

37. Ferrari E, Imbert A, Chevalier T, Mihoubi A, Morand P, Baudouy M. The ECG in pulmonary embolism. Predictive value of negative T waves in precordial leads-80 case reports. Chest 1997; 111: 537-43.

38. Kosuge M, Kimura K, Ishikawa T, Ebina T, Hibi K, Tsukahara K, et al. Prognostic significance of inverted $\mathrm{T}$ waves in patients with acute pulmonary embolism. Circ J 2006; 70: 750-5.

39. Kucher N, Walpoth N, Wustmann K, Noveanu M, Gertsch M. QR in V1-an ECG sign associated with right ventricular strain and adverse clinical outcome in pulmonary embolism. Eur Heart J 2003; 24: 1113-9.

40. Geibel A, Zehender M, Kasper W, Olschewski M, Klima C, Konstantinides SV. Prognostic value of the ECG on admission in patients with acute major pulmonary embolism. Eur Respir J 2005; 25: 843-8.

41. Calvo Romero JM, Lima Rodríguez EM. Electrocardiographic abnormalities in acute pulmonary embolism. Eur J Gen Med 2005; 2: 150-2.

42. Wolfe MW, Lee RT, Feldstein ML, Parker JA, Come PC, Goldhaber SZ. Prognostic significance of right ventricular hypokinesis and perfusion lung scan defects in pulmonary embolism. Am Heart J 1994; 127: 1371-5.

43. Ribeiro A, Juhlin-Dannfelt A, Brodin LA, Holmgren A, Jorfeldt L. Pulmonary embolism: Relation between the degree of right ventricle overload and the extent of perfusion defects. Am Heart J 1998; 135: 868-74

44. Miller RL, Das S, Anandarangam T, Leibowitz DW, Alderson PO, Thomashow $\mathrm{B}$, et al. Association between right ventricular function and perfusion abnormalities in hemodynamically stable patients with acute pulmonary embolism. Chest 1998; 113: 665-70.

45. Miniati M, Monti S, Bottai M, Scoscia E, Bauleo C, Tonelli L, et al. Survival and restoration of pulmonary perfusion in a long-term follow-up of patients after acute pulmonary embolism. Medicine (Baltimore) 2006; 85: 253-62.

46. Miller GA, Sutton GC, Kerr IH, Gibson RV, Honey M. Comparison of streptokinase and heparin in treatment of isolated acute massive pulmonary embolism. Br Med J 1971; 2: 681-4.

47. Aujesky D, Roy PM, Guy M, Cornuz J, Sanchez O, Perrier A. Prognostic value of D-dimer in patients with pulmonary embolism. Thromb Haemost 2006; 96: 478-82. 